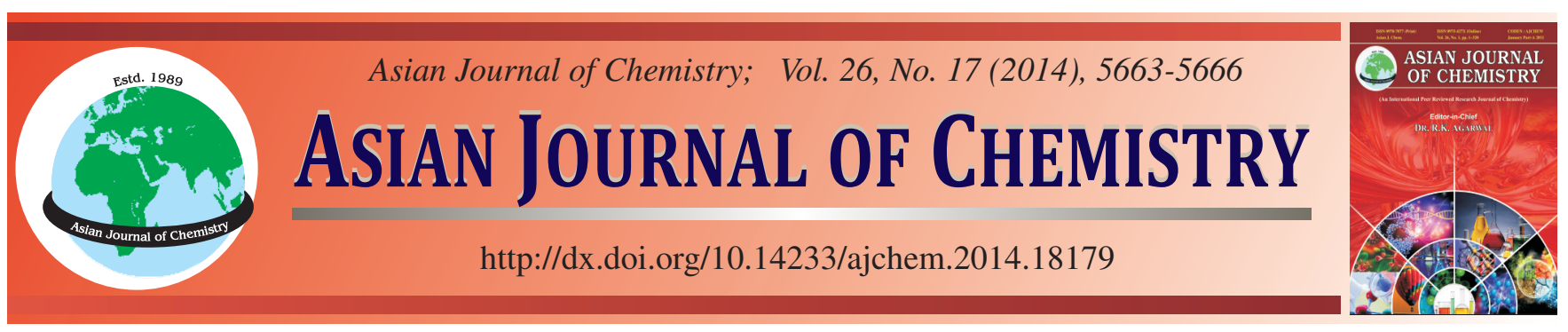

\title{
Investigation on Wear Resistance of Coated Cutting Tools Based on 17-4PH Stainless Steel Cutting Tests $\dagger$
}

\author{
ZhANFENG LiU* and Zhe WANG
}

School of Mechanical Engineering, Xi'an Shiyou University, Xi'an 710065, Shaanxi Province, P.R. China

*Corresponding author: Tel: +86 13572896975; E-mail: lzhfg9@163.com

Based on the theoretical study of cutting tool wear and the systematic analysis of the parameters affecting the wear resistance of the tools, this paper presents an investigation on the effect of coating layers on the wear resistance of cutting tool inserts. In this project, four coated cutting tool inserts were selected for the 17-4PH stainless steel cutting tests and the wear morphology and life span of the tools were studied by a scanning electron microscope (SEM) and energy spectrum analysis. According to the results, the TiAIN coating layer by the physical vapor deposition coating process displays optimal thermal stability, high resistance to oxidation even at elevated temperatures and superior durability with difficult-to-cut materials. Conversely, the other three samples exhibited relatively poor performance due to the differences in composition and structure of the coating layers.

Keywords: Coated tool inserts, Wear resistance, Cutting tool wear, 17-4PH stainless steel.

\section{INTRODUCTION}

The stainless steel referred to as $17-4 \mathrm{PH}(0 \mathrm{Cr} 17 \mathrm{Ni} 4 \mathrm{Cu} 4 \mathrm{Nb})$ is a martensitic precipitation-hardening stainless steel. With low carbon levels and high $\mathrm{Cr}$ and $\mathrm{Ni}$ content, this material exhibits good welding performance and corrosion resistance. It also contains relatively high alloy elements such as $\mathrm{Nb}$ and $\mathrm{Cu}$, which can precipitate the age-hardening stage during the heat-treatment, resulting in high strength and hardness. A few of the mechanical properties of this material are shown in Table-1.

\begin{tabular}{cc} 
TABLE-1 \\
MECHANICAL PROPERTIES OF 17-4PH STAINLESS STEEL \\
\hline Yield strength (MPa) & 772 \\
Tensile strength (MPa) & 999 \\
Elongation (\%) & 16 \\
Percentage reduction of area (\%) & 45 \\
Hardness (HRC) & 30 \\
\hline
\end{tabular}

According to Table-1, 17-4PH stainless steel presents various advantages such as high strength, excellent wear and corrosive resistance and good performance at extreme temperatures. In view of these advantages, it has been widely applied in chemical, oil, aeronautics, astronautics, nuclear industries and other fields. However, 17-4PH stainless steel is a typical difficult-to-cut material with high toughness and thermal strength, low thermal coefficient with severe work hardening and plastic deformation. Additionally, due to a considerable amount of heat released in the cutting process and the difficulty of dissipating this heat, the insert temperature is extremely high, which results in cutting scrap adhesion to the insert. This causes built-up edges which lead to increased tool wear, sample surface roughness and difficulty in cutting. To enhance the cutting capability of 17-4PH stainless steel, this paper conducted an investigation of various factors in the failure of coated cutting tools. Based on these factors, measures were taken to lower the tool wear and increase its working life ${ }^{1}$.

\section{EXPERIMENTAL}

Coated tool inserts for experiment: Generally, layers deposited by physical vapor deposition (PVD) are based on TiAlN, while those done by chemical vapor deposition (CVD) typically have multi-layered structures ${ }^{2}$. In this experiment, four coated cutting tool inserts (US735, PC9030, NC3020 and YBM251) from Mitsubishi, Korloy and ZCCCT (Zhuzhou Cemented Carbide Cutting Tools Co. Ltd) were selected and labelled as M1, M2, M3 and M4, respectively. The structures and coating processes of these samples are summarized in Table- $2^{3,4}$. 
TABLE-2

STRUCTURES AND COATING PROCESSES OF EXPERIMENTAL SAMPLES

\begin{tabular}{cccccc}
\hline Code & Model & Groove profile & Specifications & Composition & Coating process \\
\hline M1 & AC630M & EX & & TiCN $+\alpha-\mathrm{Al}_{2} \mathrm{O}_{3}$ & Chemical vapour deposition \\
M2 & PC9030 & HM & WNMG080408 & TiAlN & Physical vapour deposition \\
M3 & US735 & MA & & Multi-layered Ti compound & Physical vapour deposition \\
M4 & YBM251 & EM & & TiCN $+\mathrm{Al}_{2} \mathrm{O}_{3}+$ TiN & Chemical vapour deposition \\
\hline
\end{tabular}

Tools and characterization equipment: Tool specifications: Cw6163 engine lather, Dalian Machine Tool Group Crop.

Characterization equipment: Scanning electron microscope (SEM): JSM-6390A, Japan Electron Optics Laboratory Co., Ltd. Large tool microscope: JGX-2, Suzhou Aoka Optical Instrument Co.,Ltd.

Experimental methods: In brief, the four coated cutting tool inserts were placed in a cylindrical rotator for $17-4 \mathrm{PH}$ stainless steel. In this process, measurements of the flank wear were taken every five minutes from 0-30 min and the abrasion of the tools was captured by SEM. Additionally, the resultant wear morphologies of the different samples were observed and the effects of tool insert wear on the morphology of cutting scraps were analyzed. Based on that, the mechanisms of tool wear were investigated and the wear resistances of different coatings were studied. The parameters in the cutting process are: cutting speed $\mathrm{Vc}=120 \mathrm{~m} / \mathrm{min}$, load $\mathrm{f}=0.25 \mathrm{~mm} / \mathrm{r}$ and ap $=2 \mathrm{~mm}$.

\section{Analysis of tool wear}

\section{Wear appearance of tool inserts}

(a) Rake faces: According to Fig. 1, hard spot scratches, abrasion brightness and the exfoliation of coating layers are the main problems for the rake faces of all the samples. Also, severe particle adhesion was observed on the surface of the samples. For sample M1, obvious coating exfoliation, tiny scratches on the cutting edge, brightness in the abrasive area and particle adhesion were observed. For sample M2, severe particle adhesion was observed while the tool insert wear was relatively weak. For sample M3, extensive bright areas and obvious abrasions in the tool insert were observed (the hard alloy of the insert can be observed due to abrasion and coating exfoliation). For sample M4, slight coating abrasion was detected and obvious coating exfoliation in the tool insert area was observed.

(b) Flank faces: As shown in Fig. 2, sample M1 showed typical wear appearance with a smooth cutting edge surface, several grooves in the flank face and micro-chipping of the minor cutting edge. On sample M2, obvious particle adhesion on the cutting edge was observed, while scratches were not observed on the flank surface. For sample M3, severe coating exfoliation and large-sized scratches on the flank surface were observed, as well as breakage of the cutting edge at the maximum cutting depth. For sample M4, the appearance was similar to sample M1 (obvious scratches) and a large bright area was observed.

\section{Wear analysis}

Abrasive wear: Due to the high strength and hardness of 17-4PH stainless steel, abrasions and scratching by the hard spots on the steel may occur in the cutting process, resulting
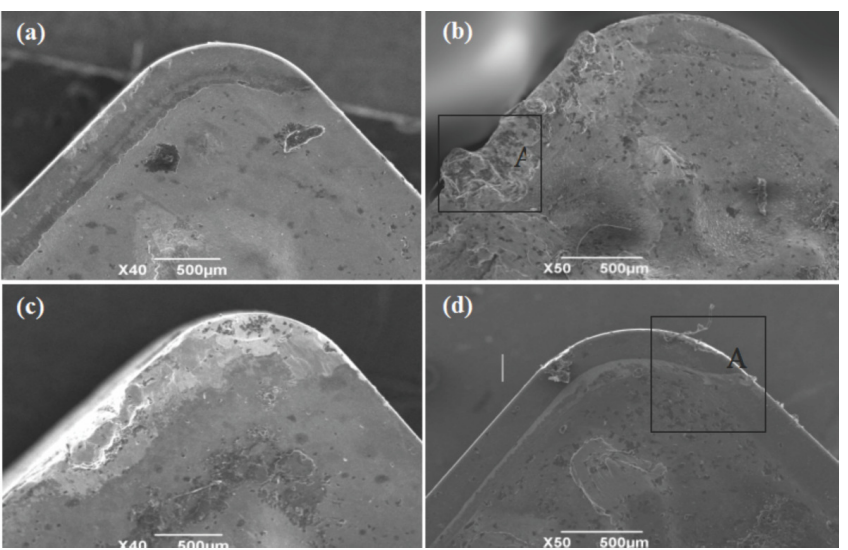

Fig. 1. Wear appearance of tool insert rake faces after cutting of 17-4PH SS: (a) M1; (b) M2; (c) M3; (d) M4

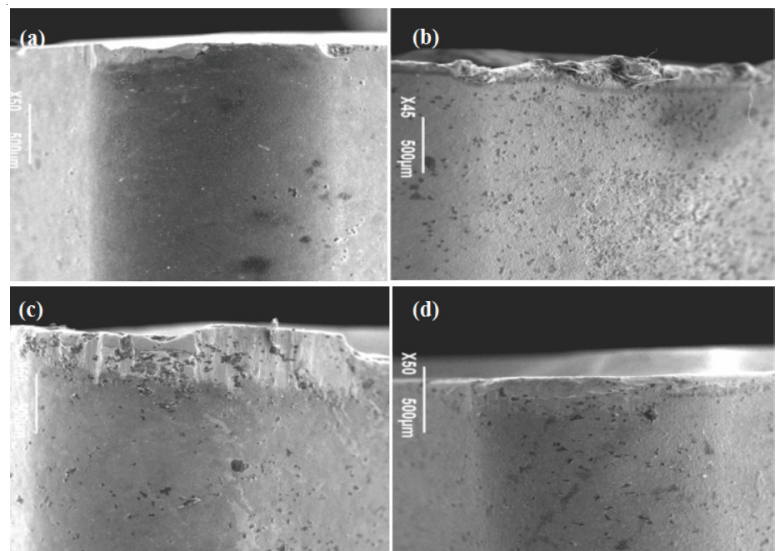

Fig. 2. Wear appearance of tool insert flank faces after cutting of 17-4PH SS: (a) M1; (b) M2; (c) M3; (d) M4

in obvious abrasive notches on the flank face called abrasive wear. In most cases, abrasive wear is inevitable and will be observed during the entire cutting process.

(b) Oxidation and diffusion wear: In the presence of heat, coating materials with low oxidation temperatures may be oxidized in the atmosphere and a film with significantly low hardness and strength is formed on the surface. As the temperature increases, the oxidation layer expands and exfoliates as a result of the difference in the linear expansion coefficient between the coating layer and the base. This is supported by Fig. 3, which shows the coating layer exfoliation in section A of sample M4 was severe.

After the coating layer exfoliation due to oxidation, carbide alloy substrate was exposed and scratched by the work piece and scrap. Furthermore, oxidation wear was also observed on the carbide alloy substrate, resulting in a bright tool insert area.

In most cases, oxidation wear is accompanied by diffusion wear. At elevated temperatures, $\mathrm{Fe}$ and $\mathrm{C}$ atoms in the work piece will diffuse into the cutting tool, while $\mathrm{W}$ and $\mathrm{C}$ atoms 


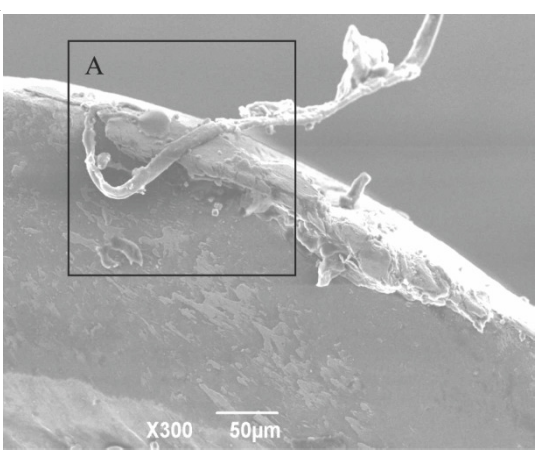

Fig. 3. Coating layer exfoliation in sample M4

in the cutting tool will diffuse into the work piece. Due to this atom exchange, the bond between atoms in cutting tools is weakened, which has a negative effect on the wear resistance of the cutting tool.

According to the energy spectrum shown in Fig. 4(a), the content of $\mathrm{W}$ and $\mathrm{C}$ are relatively high, indicating that the carbide alloy substrate has been exposed, as WC is a key component in the carbide alloy. Also, high content of $\mathrm{Fe}$ and $\mathrm{Ni}$ were observed, which is similar to gun steel. This can be explained by the scratching of the exposed alloy by the work piece and resultant particle adhesion on the surface of the cutting tool. Besides, the high content of $\mathrm{Fe}$ and $\mathrm{Ni}$ can also be explained by mutual diffusion of atoms between the cutting tool and the work piece.
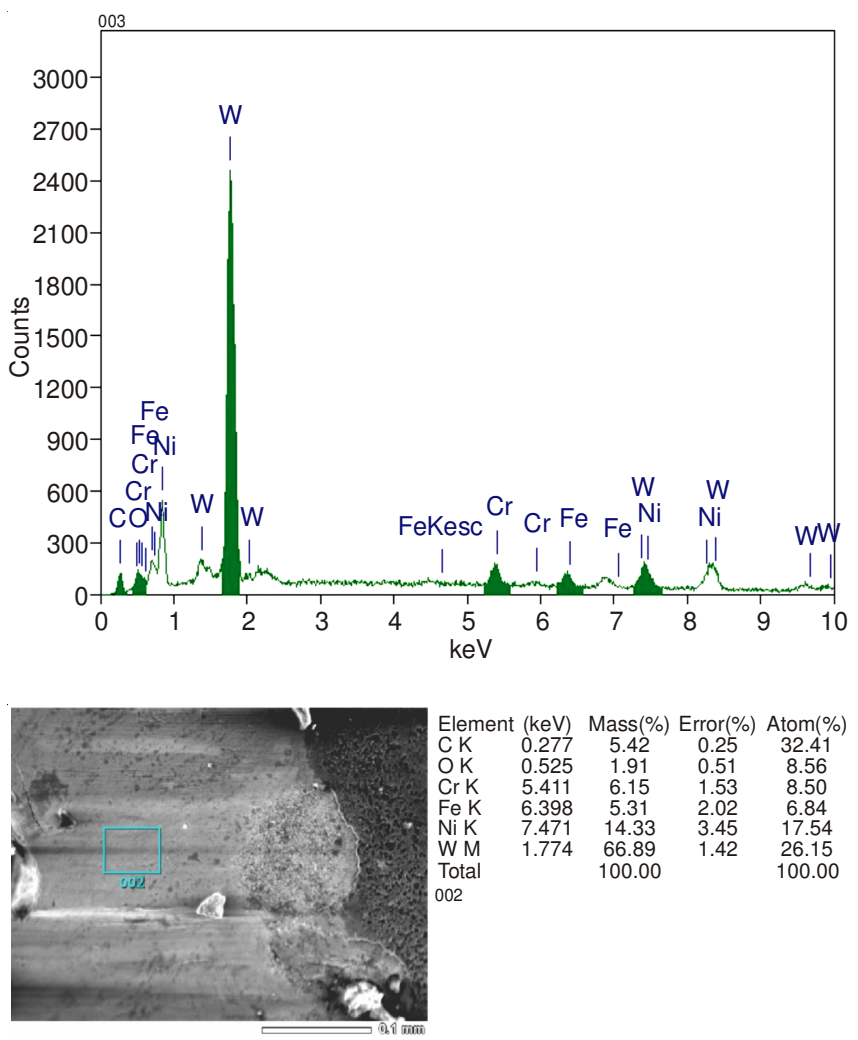

Fig. 4. (a) Energy spectrum of the flank face; (b) sectional element analysis

Additionally, the energy spectrum revealed that the oxygen content was also high, which can be explained by oxidation wear on the surface of the carbide alloy as a result of its oxidation at elevated temperatures.
Adhesive wear: According to the energy spectrum shown in Fig. 5(a) below, contents of metal elements such as $\mathrm{Fe}, \mathrm{Cr}$ and $\mathrm{Cu}$ are high and this is inconsistent with the composition of 17-4PH stainless steel, indicating that the adhesion particles were from the work piece. In addition, the high content of oxygen indicates that the adhered particles were oxidized at elevated temperatures.
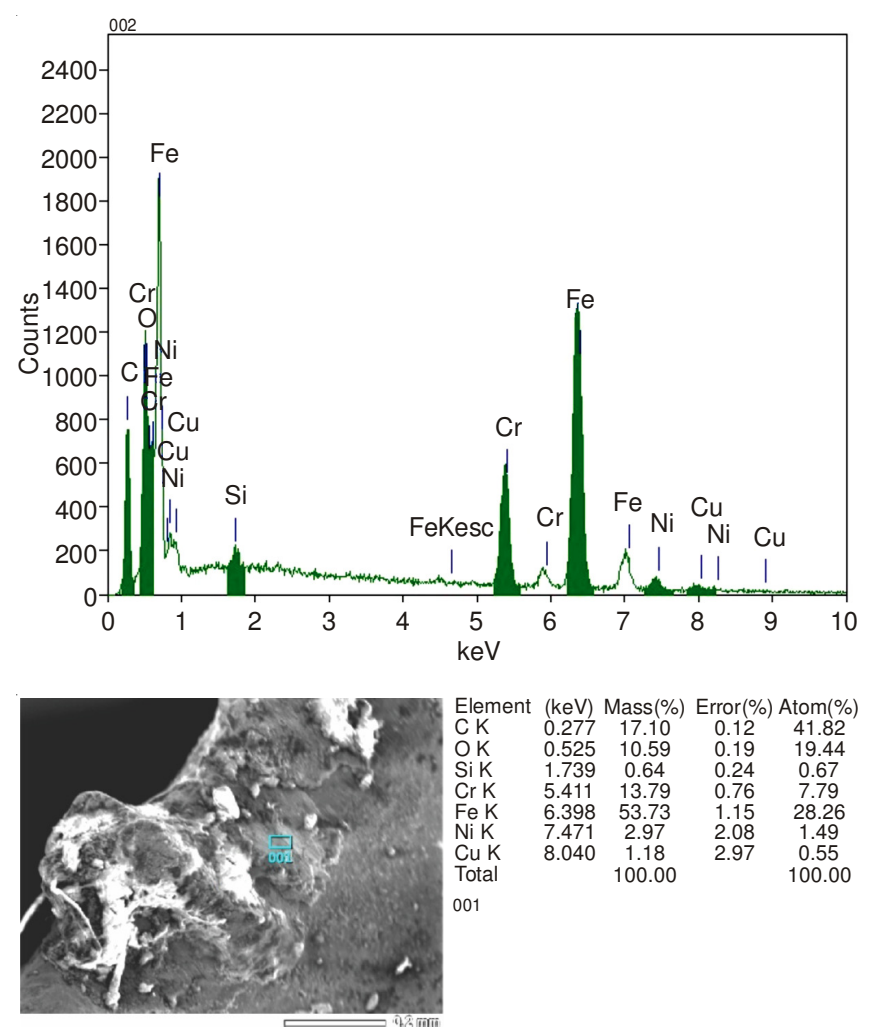

Fig. 5. (a) Energy spectrum of the rake face; (b) sectional element analysis

The reason for adhesive wear is the affinity of stainless steel to the cutting tools. In the cutting process, surface contact between the tool and the work piece due to roughness resulted in adhesion on both surfaces at a certain temperature and pressure. Also, adhesion of cutting scraps can lead to built-up edges, which may exacerbate the adhesive wear as a result of the relative motion between the cutting tool and the work piece. Particles on the tool insert will peel off (micro chipping) due to the presence of scraps, built-up edges and hard spots on the work piece.

\section{Analysis of wear resistance of different coatings}

Effects of coating materials: In this experiment, sample M1 was coated by $\mathrm{Al}_{2} \mathrm{O}_{3}$, which has high hardness, excellent chemical stability and oxidation resistance at elevated temperatures. As a result, sample M1 showed superior resistance to oxidation and crater wear and thermoplastic deformation. Nevertheless, coating exfoliation and bright areas caused by oxidation wear were observed.

Sample M2 was coated by TiAlN and presented the best wear resistance among all the samples tested. This can be explained by the superior thermal stability and oxidation resistance at elevated temperatures. In fact, a film of $\mathrm{Al}_{2} \mathrm{O}_{3}$ was formed as a result of the reaction between $\mathrm{Al}$ and $\mathrm{O}_{2}$ in the atmosphere and this compact film prevented further oxidation. For this 
reason, oxidation wear was much weaker compared to the other samples. However, adhesion was observed because of the weak adhesion resistance of TiAlN.

Sample M3 was coated with a titanium compound with $\mathrm{Ti}, \mathrm{C}$ and N. In virtue of the high hardness of TiC and TiCN, Sample M3 showed the highest hardness among all the samples, thus having excellent initial wear resistance (at low temperatures). However, the coating layer was rapidly oxidized as the temperature increased, resulting in severe oxidation wear.

Sample M4 was coated by TiN. This material presented low hardness and poor wear resistance in the beginning, as its flank face wear was much higher than other samples. Nevertheless, TiN showed superior adhesion resistance ; no adhesion was observed for sample M4.

Effect of coating structures and processes: In this experiment, coatings of samples M1, M3 and M4 were multilayer structures by chemical vapour deposition (CVD), while that of sample M2 was a single-layer structure by physical vapour deposition (PVD).

For sample M1, the substrate was initially coated with TiCN, followed by a thin layer of $\mathrm{Al}_{2} \mathrm{O}_{3}$ and finished with another layer of TiCN. By altering the content of $\mathrm{C}$ and $\mathrm{N}$ continuously, a multi-layer structure was developed, which resulted in lower internal stress and therefore, better cohesion. For this reason, this material was used as the first and innermost coating layer. The sandwiched $\mathrm{Al}_{2} \mathrm{O}_{3}$ coating layer between the two TiCN layers showed excellent thermal insulation (to prevent over-heating of the substrate), as well as superior hightemperature behaviours. With all the factors mentioned above, sample M1 showed excellent overall performance in the experiment.

For sample M3, the multi-layer structure significantly increased the interface area and decreased grain size. Also, the relatively low residual stress effectively enhanced the adhesion, hardness and toughness of the coating layers.

For sample M4, the double-layer structured coating was achieved with TiCN as the first layer and a thick layer of $\mathrm{Al}_{2} \mathrm{O}_{3}$ as the second one. According to the experimental results, this coating showed similar wear resistance but slightly longer working life compared to Sample M1. With a thick layer of $\mathrm{Al}_{2} \mathrm{O}_{3}$, this coating showed excellent thermal insulation at elevated temperatures.

For sample M2, the coating was a single layer of TiAlN applied by physical vapor deposition. This coating showed lower thickness and weaker adhesion to the substrate, compared to those done by chemical vapor deposition. Theoretically, wear resistance should increase with an increase in coating layer thickness, but this was not the case. One possible explanation is the increasing residual stress, which may lower the strength of the layer and result in cracks.

Generally, coating done by physical vapor deposition results in better wear resistance compared to one done by chemical vapor deposition. Among the chemical vapor deposition coated layers, sample $\mathrm{M} 1$ with a thick layer of $\mathrm{Al}_{2} \mathrm{O}_{3}$ showed the best wear resistance, followed by sample M4 with a triple-layer structure, while Sample M3 with a multi-layer structure coating of Ti compound showed inferior wear resistance, especially at elevated temperatures.

\section{Conclusion}

- According to the investigation on the wear mechanism and factors involved, the characteristics of the coating material are proved to be the determining factor, as long as reasonable cutting parameters were applied.

- According to the SEM images and EDS spectrum, severe adhesive wear, apart from abrasive, oxidation and diffusion wear, was observed in the 17-4PH stainless steel cutting test. This is believed to be related to the adhesive properties of this stainless steel.

- In the experiment, indexable inserts coated with four different coatings were tested and analyzed. It was shown that TiAlN by physical vapor deposition presented excellent high-temperature oxidation resistance and stability, but poor adhesion and diffusion wear resistance compared to coatings done by chemical vapor deposition. This coating should be applied in the semi-finish process of difficult-to-cut materials. Multi-layered Ti compound coating led to high hardness but poor oxidation resistance at elevated temperatures. As a result, this coating is suitable for medium/low speed machining at low temperatures. The other two coatings gave similar wear resistance, while $\mathrm{TiCN}+\mathrm{Al}_{2} \mathrm{O}_{3}$ showed better thermal stability in virtue of its thick layer of $\mathrm{Al}_{2} \mathrm{O}_{3}$. As a result, this coating led to slightly better wear resistance compared to the $\mathrm{TiCN}+\mathrm{Al}_{2} \mathrm{O}_{3}$ + TiN coating.

\section{REFERENCES}

1. T.L. Wang et al., Automotive Eng., 1, 55 (2012).

2. H. Li, B. Li and H. Hu, Tool Eng., 4, 3 (2010).

3. Mitsubishi, Samples of Mitsubishi Comprehensive, Mitsubishi Composite Materials Co. Ltd. (2012).

4. ZCCCT, Samples of Zhuzhou Diamond Cutting Tool Product, Zhuzhou Cemented Carbide Cutting Tools Co. Ltd. (2013). 